\title{
The new $\mathrm{K}_{\mathrm{L}} \rightarrow \pi^{0} v \bar{v}$ experiment (KOTO) at J-PARC
}

\author{
Hiroaki Watanabe ${ }^{1}$ for the J-PARC E14 KOTO collaboration \\ HIGH ENERGY ACCELERATOR RESEARCH ORGANIZATION, KEK \\ 1-1 Oho, Tsukuba, Ibaraki 305-0801 Japan \\ E-mail: nabe@post.kek.jp
}

The neutral-kaon decay $\mathrm{K}_{\mathrm{L}} \rightarrow \pi^{0} v \bar{v}$ is a direct CP-violating process caused by a flavorchanging neutral current, and the branching ratio is predicted to be $(2.49 \pm 0.39) \times 10^{-11}$ in the Standard Model. The rare decay is one of the processes expected to have a significant impact on new physics searches. The J-PARC E14 KOTO experiment aims at the first observation of the rare decay $\mathrm{K}_{\mathrm{L}} \rightarrow \pi^{0} v \bar{v}$ using the upgraded detector from the KEK-E391a experiment and an intense $\mathrm{K}_{\mathrm{L}}$ beam at J-PARC. The new dedicated $\mathrm{K}_{\mathrm{L}}$ beam line for the KOTO experiment was constructed in 2009 and the first $\mathrm{K}_{\mathrm{L}}$ beam was successfully extracted from October 2009 to February 2010. The construction of the KOTO detector with many upgrades was started in 2009. In this contribution, the current status of the KOTO experiment is reported.

35th International Conference of High Energy Physics (ICHEP2010)

Paris, France

July 22-28, 2010

\footnotetext{
$1 \quad$ Speaker
} 


\section{Introduction}

The neutral-kaon decay $\mathrm{K}_{\mathrm{L}} \rightarrow \pi^{0} v \bar{v}$ is a direct CP-violating process and the branching ratio of the decay is predicted to be $(2.49 \pm 0.39) \times 10^{-11}$ in the Standard Model [1]. The decay amplitude is proportional to a CKM parameter, $\operatorname{Im}(\mathrm{Vtd}) \propto \eta$, corresponding to the CP-violation amplitude in the Standard Model. Since the branching ratio can be calculated with exceptionally small theoretical uncertainties, experimental measurement of the decay precisely determines the CP-violation amplitude. Also, the decay is one of the most sensitive processes to new physics beyond the Standard model [2]. However, the experiment is very challenging because of poor kinematical signatures of single $\pi^{0}$ in the three-body decay against huge amount of background events from other kaon decays, or $\pi^{0}$ and $\eta$ production by neutron interactions.

\section{KOTO experiment}

The KOTO ( $\mathbf{K}^{\mathbf{0}}$ at Tokai, where the J-PARC is located) experiment aims at the first observation of the rare decay $\mathrm{K}_{\mathrm{L}} \rightarrow \pi^{0} v \bar{v}$ by using the upgraded KEK-E391a detector and a highintensity $\mathrm{K}_{\mathrm{L}}$ beam at J-PARC [3]. In the KEK-E391a results, the main background source was halo-neutron interactions [4]. When a neutron apart from the beam center (halo neutron) hits a detector subsystem, a $\pi^{0}$ may be produced by the interaction between the neutron and the detector materials. Thus, KOTO is designed to reduce the halo-neutron events down to five orders of magnitudes by using a new dedicated beam line and with detector upgrades, as described in later sections.

There are many new physics scenarios to predict a larger branching ratio than the Standard Model expectation; they will be examined according to the improvement of the KOTO sensitivity. After the observation of the decay, the experiment will proceed to a precise measurement of the branching ratio of the decay by collecting more than 100 events.

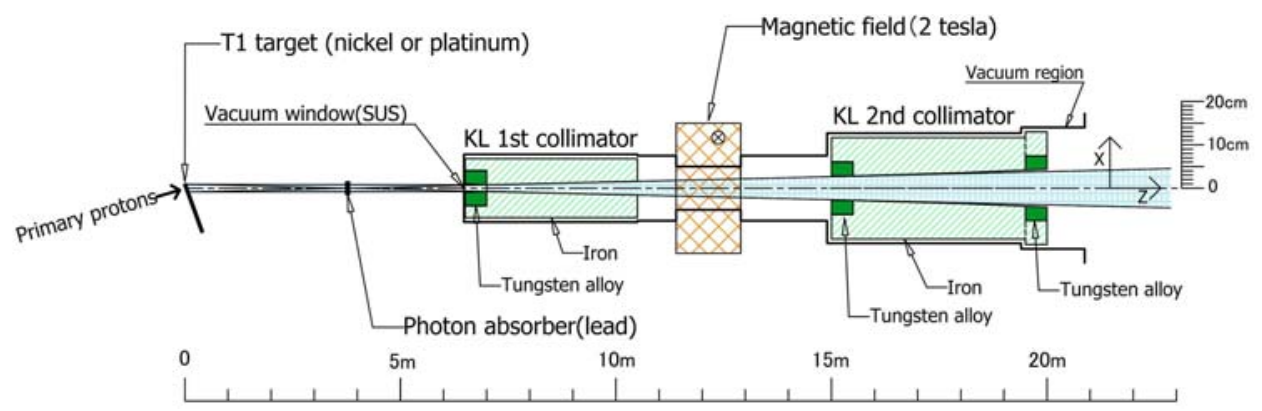

Figure 1. Schematic drawing of the $\mathrm{K}_{\mathrm{L}}$ beam line.

\section{3. $\mathrm{K}_{\mathrm{L}}$ beam line}

Figure 1 shows a schematic drawing of the beam line for the KOTO experiment. It is extracted at $16^{\circ}$ with respected to the primary proton beam. The neutral beam is collimated by two stages of long collimators made of iron and tungsten blocks. The collimation lines are designed so as to reduce the multiple scattering of neutrons, which is the main source of halo neutrons. The collimators are located inside vacuum in order to remove neutron scattering with 
air inside the beam line, and will be connected directly to the vacuum chamber which contains the detectors. Charged particles inside the beam line are removed by a dipole magnet located between two collimators. In front of the first collimator, a photon absorber made of lead is located in order to reduce the photon flux and to reduce the single counting rate of the detectors located near the beam.

Figure 2 shows the profiles of neutrons obtained by a Monte-Carlo simulation [5]. Since the effective cross section of the production target seen from the detector is rectangular, the collimation lines were optimized separately in the vertical and the horizontal planes to maximize the $\mathrm{K}_{\mathrm{L}}$ yield and minimize the halo-neutron flux. A ratio of the halo-neutron flux to the $\mathrm{K}_{\mathrm{L}}$ flux is expected to be $0.07 \%$, which is smaller than that of KEKE391 a by a factor of 240 .

The beam-line construction was completed in September 2009, and a beam survey was performed from October 2009 to February 2010. The primary goal of the survey was to measure the $\mathrm{K}_{\mathrm{L}}$ flux because there was a discrepancy, by a factor of three, among the simulation codes. Since the GEANT4 calculation shows a smaller flux than those of FLUKA and GEANT3, the KOTO sensitivity was conservatively estimated based on the GEANT4 simulation in the proposal. In the survey, the $\mathrm{K}_{\mathrm{L}}$ was measured by detecting the $\mathrm{K}_{\mathrm{L}} \rightarrow \pi^{+} \pi^{-} \pi^{0}$ decay using four

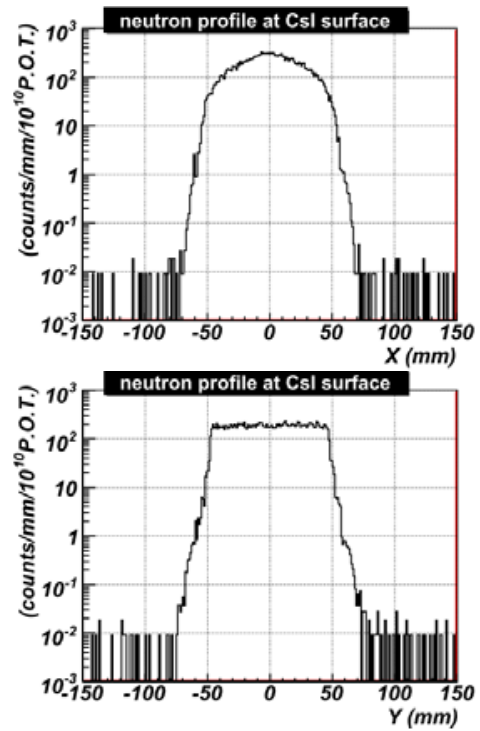

Figure 2. Neutron beam profile in the vertical (top) and horizontal (bottom) views obtained by the Monte-Carlo simulation. hodoscopes for tracking of the $\pi^{+} \pi^{-}$and two Cesium-Iodide (CsI) calorimeters for photon detections from the $\pi^{0}$ decay [6]. The $\mathrm{K}_{\mathrm{L}}$ events were clearly reconstructed as shown in Figure 3. The $\mathrm{K}_{\mathrm{L}}$ flux was obtained to be $1.83 \times 10^{7} \mathrm{~K}_{\mathrm{L}}$ 's $/ 2 \times 10^{14}$ protons-on-target (preliminary), which is 2.3 times larger than the $\mathrm{K}_{\mathrm{L}}$ flux used in our proposal. The beam profile was obtained from the counting rates of a leadtungstate (PWO) crystal moving inside the beam. The measured profiles agree well to those of the Monte-Carlo simulation including detector response.

\section{KOTO Detector}

Figure 4 shows a schematic cross-sectional view of the KOTO detector. The configuration remains to be same as the KEK-E391a detector and many parts of the detector will be reused. There are, however, considerable upgrades at KOTO in order to reject neutron backgrounds and to take data effectively in a high-intensity environment.

The first item of the upgrades is the replacement of CsI

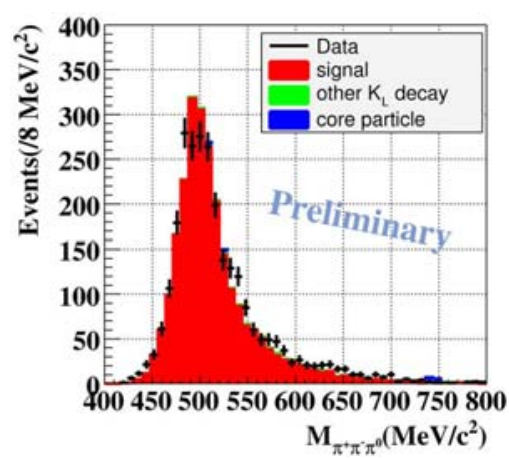

Figure 3. Comparison of the reconstructed invariant-mass distritbutions of $\pi^{+} \pi^{-} \pi^{0}$ beteween the data (dots with error bar) and the Monte-Carlo simulaiton (shaded histogram). crystals in the electromagnetic calorimeter. The new crystals which were used in the Ferimlab$\mathrm{KTeV}$ experiment are of two sizes, $2.5 \times 2.5 \times 50 \mathrm{~cm}^{3}\left(27-\mathrm{X}_{0}\right)$ for the central region $(2240$ blocks), and $5.0 \times 5.0 \times 50 \mathrm{~cm}^{3}$ for the outer region (336 blocks). These crystals are longer, and 
have smaller cross-sections compared to the KEK-E391a crystals with $7 \times 7 \times 30 \mathrm{~cm}^{3}\left(16-\mathrm{X}_{0}\right)$. By this upgrade, many improvements are expected such as better energy resolution, better reconstructed-vertex resolution with less tail due to smaller shower leakage, higher efficiency of photon detection, reduction of $2 \gamma$ 's fusion events, and better $\mathrm{n} / \gamma$ identification, as described in Ref. [3]. The outputs of the calorimeter are recorded by waveform digitizers to obtain better timing resolution in a high-rate environment.

The second is the upgrades of veto counters particularly for the $2^{\text {nd }}$ collar counter $(\mathrm{CC} 02)$, the Beam Hole Photon Veto (BHPV) and the Main-Barrel Counter (MB). The CC02 is one of the main background sources in the KEK-E391a experiment. The CC02 will be moved to upstream and replaced from the lead-scintillator sandwich modules to segmented CsI crystals. By the CC02 upgrades, halo-neutron background associated with the $\mathrm{CC} 02$ is expected to be reduced by a factor of 20. The BHPV counters detect extra photons in the $\mathrm{K}_{\mathrm{L}}$ decay escaping through the beam hole and have to be insensitive to neutrons keeping high detection efficiency for the photons. The BHPV is designed to be an array of aerogel Cherenkov counters with lead convertors. Due to Cherenkov threshold effects and longitudinal shower-profile differences between the neutron and the photon, an over-veto rate can be suppressed to be $3 \%$ level. Finally, the $\mathrm{MB}$, which is the largest veto counter surrounding the decay volume, will be upgraded. By adding additional 5 radiation lengths in the thickness, the photon-detection inefficiency due to punch-through will be negligible. By the detectors upgrades together with the beam-line optimization, halo-neutron background is expected to be negligible small.

We plan to complete the construction of the electromagnetic calorimeter with full read-out system in 2010. An engineering run with the new calorimeter will be performed just after the construction. The KOTO detector will be completed within 2011, and the first physics run will be carried out by the summer of 2012. Since the beam intensity at J-PARC is expected to be $10 \%$ of designed value at that time, the first KOTO sensitivity will be $1 \times 10^{-9}$ after one month of data taking, which is already better than the indirect "Grossman-Nir" limit $\left(1.2 \times 10^{-9}\right)$ evaluated from the branching ratio of the $\mathrm{K}^{+} \rightarrow \pi^{+} v \bar{v}$ decay using the Isospin symmetry.

\section{References}

[1] F. Mescia and C. Smith, Phys. Rev. D 76, 034017 (2007).

[2] http://www.lnf.infn.it/wg/vus/ content/Krare.html.

[3] Proposals for Nuclear and Particle Physics Experiments at J-PARC,

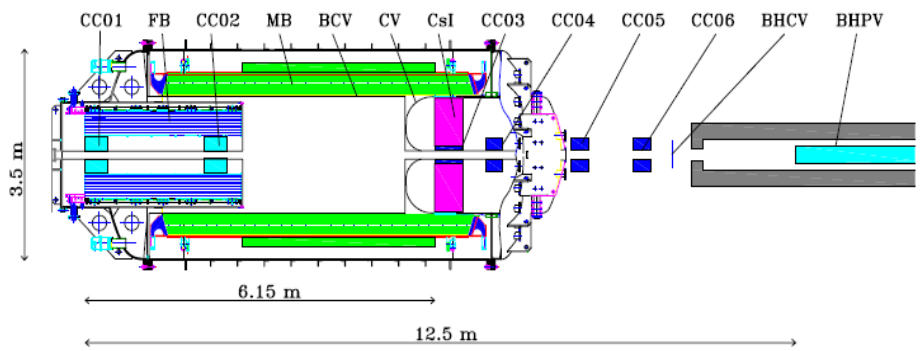

Figure 4. Cross-sectinal view of the KOTO detector. http://j-parc.jp/NuclPart/Proposal_e.html.

[4] J. K. Ahn et al., Phys. Rev. D 81, 072004 (2010), and references therein.

[5] T. Shimogawa, Nucl. Instrum. Meth. Phys. Res. A 623, (2010) 585-587.

[6] K. Shiomi, to appear in these proceedings. 